Energy Research Journal 1 (1): 36-41, 2010

ISSN 1949-0151

(C) 2010 Science Publications

\title{
Photostability of Ponceau 2R Doped Thin Film Sol-Gel Silica as Luminescent Solar Collector
}

\author{
S.M. Reda \\ Department of Chemistry, Faculty of Science, Benha University, Benha, Egypt
}

\begin{abstract}
Problem statement: Current researches had shown that the photostability of a luminescent solar collector depend on the method of its preparation and type of using dye. This research therefore aimed at studying the stability of luminescent solar collector prepared by sol-gel spin coating method using Ponceau 2R. Approach: The effect of silica matrix on the photostability and fluorescence properties of Ponceau 2R was investigated in terms of concentration using absorption and emission techniques. The photostability of Ponceau 2R doped sol-gel samples was also examined through 60 days exposure to sunlight. Results: The dye in the solid matrix had absorption and emission characteristics different from those in the liquid solution. The transition energy for electrons were direct allowed transition for all examined concentrations and there is no variation in the values of optical gap Energy $\left(\mathrm{E}_{\mathrm{g}}\right)$ by increasing the dye content or exposure times. The dependence of the width of the tails of the localized states in the band gap $\left(\mathrm{E}_{\mathrm{u}}\right)$ on the concentration and exposure times were also studied. The emission spectra for the samples before and after exposure to sunlight showed that the sample of concentration $0.1 \mathrm{wt} \%$ had the highest fluorescent intensity and fluorescence quantum yield. Conclusion/Recommendations: This study showed that Ponceau 2R of $0.1 \mathrm{wt} \%$ concentration has a good potential for application in a luminescent solar collector using sol-gel techniques.
\end{abstract}

Key words: Luminescent solar collector, sol-gel, ponceau 2R, optical gap energy, photostability, fluorescence quantum yield

\section{INTRODUCTION}

Solar energy can be considered to solve the energy problem if appropriate methods are developed to collect, concentrate and convert solar radiation. Luminescent Solar Collectors (LSCs) have been used to collect and concentrate solar radiation (Earp et al., 2004). They have several advantages over geometric concentrators in that solar tracking is unnecessary, both diffuse and direct radiation are concentrated and the heat dissipation problems are reduced (Debije et al., 2009). A luminescent solar concentrator consists of a transparent flat plate containing luminescent particles such as organic dyes, inorganic phosphors or recently quantum dots (Gallagher et al., 2007). Sunlight is absorbed by luminescent species and then the luminescent is trapped in the plate by total internal reflection and concentrated at the edges of the plate. A solar cell can be chosen to convert the luminescent trapped light at the edge, as shown in Fig. 1. Many designs are fabricated for the LSCs, such as doped polymer sheet and sol-gel thin films on glass (Bailey et al., 2007). The use of the solgel technique to produce thin glass coating was applied to develop materials for application in the solar energy systems (Pettit and Brinker, 1986). The advantages of the sol-gel process are lower temperature processing, low cost and the possibility of incorporating organic molecules at low temperature which destroyed in conventional glass-forming process (Abbas et al., 2005). The silica matrix may be used as a host for several dyes (Maruszewski, 1999), due to such internal properties as transparency, chemical inertness and mechanical resistance (Laranjo et al., 2007). The advantage of doped thin films rather than bulk doped plates is that the luminescence emitted from the thin film is trapped in the plate while parasitic losses due to self-absorption and scattering from impurities can be reduced (Reisfeld et al., 1994). Among the dyes which are used in the preparation of luminescent solar concentrator, the Azo dyes are important colorants and constitute the largest class of dyes having extensive application in textiles, papers, leathers, additives, foodstuffs and cosmetics (Gnanamani et al., 2005). Additionally, this type of dye can act as dye lasers and could be applied in nonlinear optics (Costa et al., 2004). Within the ensemble of Azo type dyes, one important molecule is Ponceau 2R, due to its great photostability and high quantum yield in fluorescence emission. 


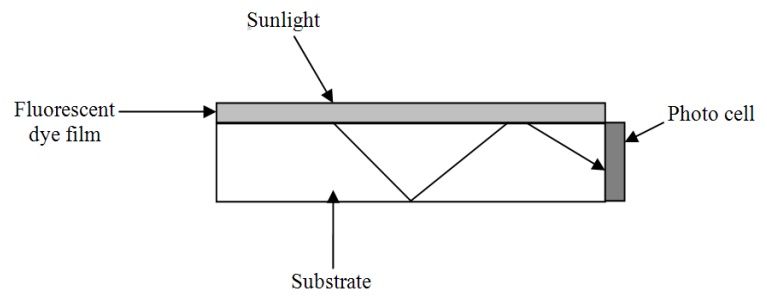

Fig. 1: Thin film luminescent solar collector

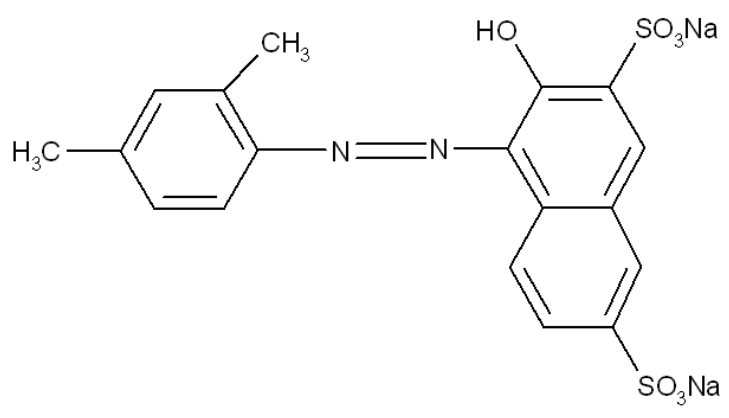

Fig. 2: The molecular structure of Ponceau 2R

Therefore, there is a great interest in the incorporation of this type of dye in a silica matrix. The molecular structure of Ponceau 2R is shown in Fig. 2.

In this study sol-gel spin coating was used to prepare thin films of silica gel doped with Ponceau 2R. The effect of the silica matrix on the photostability and fluorescence properties of this dye was studied in order to verify the potential use of this kind of dye for LSC applications.

\section{MATERIALS AND METHODS}

The silica-gel samples doped with Ponceau 2R (Fluka) were prepared by hydrolysis and polycondensation of Tetraethylorthosilicate (TEOS) in water and ethanol solutions under acidic conditions. The initial solution contained $7 \mathrm{~mL}$ of TEOS (Aldrich), $7 \mathrm{~mL}$ of ethyl alcohol, $8 \mathrm{~mL}$ of water and $1 \mathrm{~mL}$ of $\mathrm{HCl}$ serving as catalyst. Ponceau $2 \mathrm{R}$ was added to the initial solution under magnetic stirring for $1 \mathrm{~h}$ at $60^{\circ} \mathrm{C}$. Thin films were produced by coating glass slides with the above solution by spin coating at a constant speed $900 \mathrm{rpm}$. Subsequently, the samples were dried at $60^{\circ} \mathrm{C}$ for $5 \mathrm{~min}$. Plate samples of thickness $(\simeq 0.05 \mathrm{~cm})$ and dye concentrations of $(0.1,0.15$ and $0.2 \mathrm{wt} \%)$ were prepared in the sol-gel matrix.

The outdoor testing was carried out for 60 days, starting in July in summer of 2006 in National Institute of Laser, Cairo University, Giza, Egypt. The absorption spectra were measured with a Perkin-Elmer Lambda 40 spectrophotometer and emission spectra were recorded on a Jasco FP777 (Japan) spectrofluorometer.

\section{RESULTS AND DISCUSSION}

Optical properties: The absorption spectra for Ponceau 2R in ethanol solution and in sol-gel thin film are shown in Fig. 3. From Fig. 3 we can see that, the peak wavelength of absorption of Ponceau $2 R$ in solid matrix shows a blue shift from that in the liquid media. This shift of the absorption maximum of the dye upon incorporation might be caused by solvent effects induced by the silica matrix (Maruszewski, 1999). Also we notice that the absorption band width of Ponceau $2 \mathrm{R}$ is broader in solid matrix than in liquid medium. This broadening may be attributed to the influence of the host on the dye agglomeration (Maruszewski, 1999).

Figure 4 shows that for all the three concentrations $(0.1,0.15$ and $0.2 \mathrm{wt} \%)$, are taken for study the shape of the dye's absorption spectrum is independent of the concentration over the range examined of dye's absorbance. The absorption spectrum of the undoped silica glass film is also examined as shown in Fig. 5. From Fig. 5 we noticed that the undoped sample is transparent over range of wavelengths. So that the absorption spectrum detected is only from the dye under examination.

The photostability of Ponceau 2R doped sol-gel: The photostability of Ponceau $2 \mathrm{R}$ in sol-gel thin film under outdoor exposure to sunlight at different times was studied. The results show that, for all examined concentrations, the optical density of dyes upon exposure to sunlight is decreased by increasing time of exposure, Fig. 6. The observed decrease in absorbance value can be attributed to photochemical decomposition of Ponceau $2 \mathrm{R}$ as a result of absorption of the solar radiation.

The kinetics of the photodegradation of Ponceau $2 \mathrm{R}$ samples through exposure to sunlight have been studied by following the change in the intensity of the absorption at their absorption maxima. The degradation process was found to obey a first- order kinetic equation in which:

$\ln \frac{A_{o}}{A_{t}}=k t$

Where:

$\mathrm{k}=$ The first rate constant

$\mathrm{A}_{\mathrm{o}}$ and $\mathrm{A}_{\mathrm{t}}=$ The absorbance at time $\mathrm{t}=0$ and $\mathrm{t}=\mathrm{t}$ respectively 


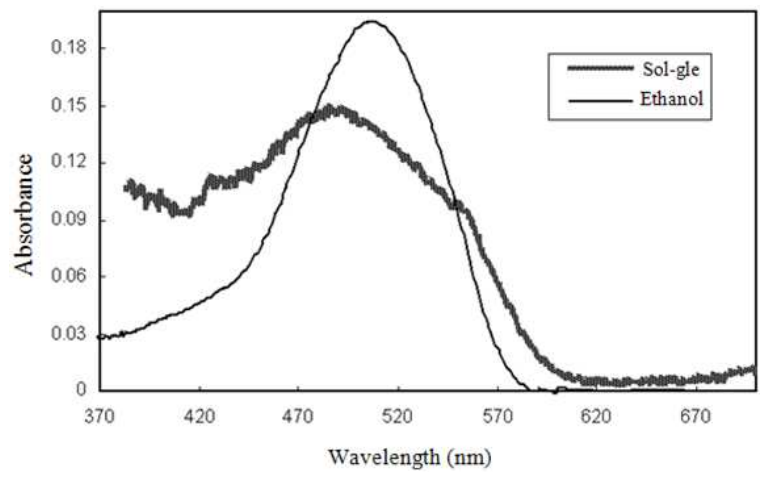

Fig. 3: Absorption spectra of Ponceau 2R in ethanol and the doped sol-gel glass film

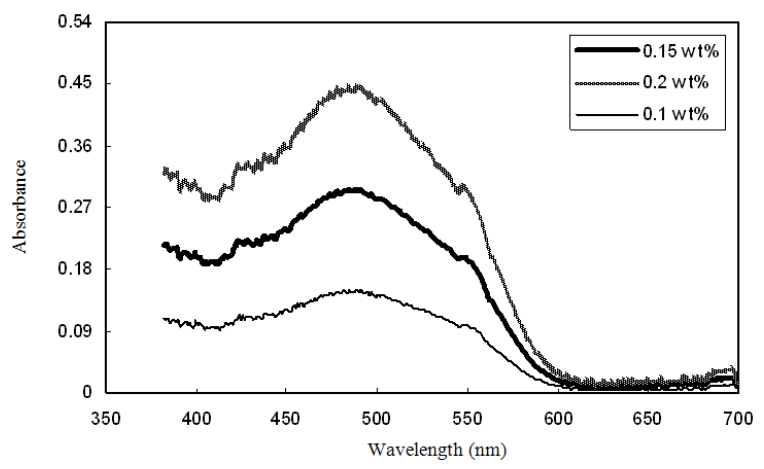

Fig. 4: Absorption spectra for various concentrations of Ponceau 2R in sol-gel glass films

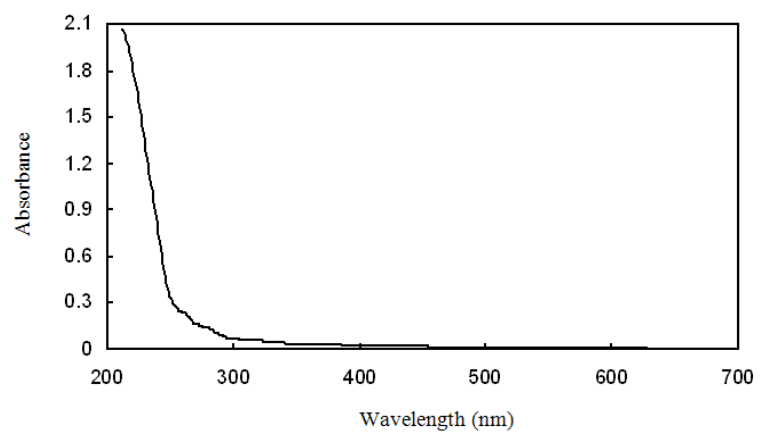

Fig. 5: The absorption spectra of undoped silica glass film

The first-order rate equation plots $\ln \left(\mathrm{A}_{\mathrm{o}} / \mathrm{A}_{\mathrm{t}}\right)$ Vs $\mathrm{t}$ for degradation of Ponceau $2 \mathrm{R}$ samples are given in Fig. 7. From the slope of these curves the rate of photodegradation was calculated and listed in Table 1. The lower degradation rate of concentration $0.1 \mathrm{wt} \%$ refers to the possibility of using this concentration to prepare some solar collectors.

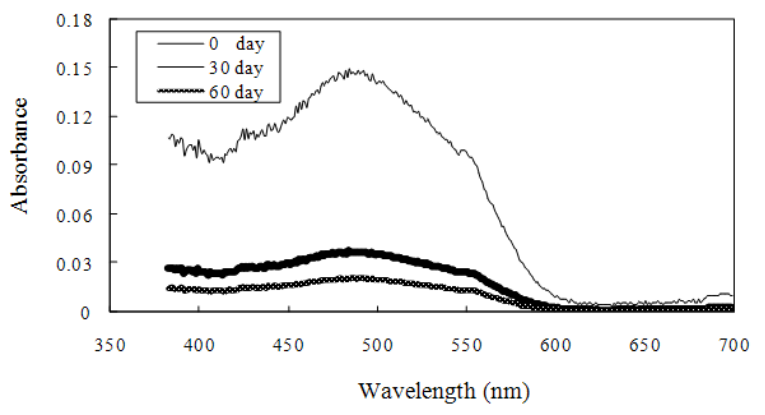

Fig. 6: The change in the absorbance for $0.1 \mathrm{wt} \%$ of Ponceau $2 \mathrm{R}$ in sol-gel glass film upon exposure to sunlight at different times

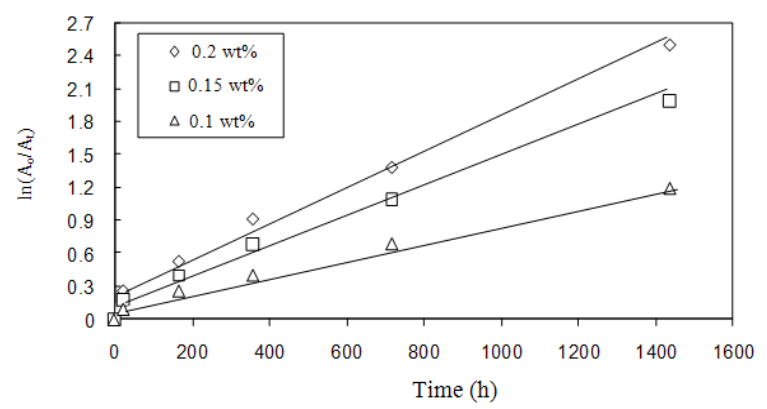

Fig. 7: The first-order equation plots for degradation of various concentrations of Ponceau $2 \mathrm{R}$ in sol-gel glass film upon exposure to sunlight

Table 1: The optical parameters $\left(E_{g}\right.$ and $\left.E_{u}\right)$ and specific rate of degradation for Ponceau 2R samples in sol-gel glass films before and after exposure to sunlight

\begin{tabular}{|c|c|c|c|c|}
\hline $\begin{array}{l}\text { Concentration } \\
\text { (wt } \%)\end{array}$ & $\begin{array}{l}\text { Exposure } \\
\text { time (day) }\end{array}$ & $\begin{array}{l}{ }^{\mathrm{a}} \mathrm{E}_{\mathrm{g}} \\
(\mathrm{eV})\end{array}$ & $\begin{array}{l}{ }^{b} E_{u} \\
(e V)\end{array}$ & $\begin{array}{l}{ }^{c} \mathrm{k} \times 10^{2} \\
\left(\mathrm{~h}^{-1}\right)\end{array}$ \\
\hline \multirow{2}{*}{0.10} & 0 & 2.05 & 0.08 & 0.07 \\
\hline & 60 & 2.05 & 0.07 & \\
\hline \multirow[t]{2}{*}{0.15} & 0 & 2.05 & 0.11 & 0.11 \\
\hline & 60 & 2.05 & 0.08 & \\
\hline \multirow[t]{2}{*}{0.20} & 0 & 2.05 & 0.14 & 0.13 \\
\hline & 60 & 2.05 & 0.11 & \\
\hline
\end{tabular}
constant

For thin film materials the absorption coefficient $(\alpha)$ at angular frequency $(\omega)$ of radiation can be calculated using the formula (Mansour, 2004):

$\alpha(\omega)=2.303 \frac{\mathrm{A}}{\mathrm{x}}$

Where:

$\mathrm{x}=$ The sample thickness

$\mathrm{A}=$ The optical absorbance 
At high absorption levels the optical gap energy $\left(\mathrm{E}_{\mathrm{g}}\right)$ of the system related to the absorption coefficient by (Mansour, 2004):

$$
\alpha \mathrm{E}=\left(\mathrm{E}-\mathrm{E}_{\mathrm{g}}\right)^{\mathrm{n}}
$$

Where:

$$
\begin{array}{ll}
\mathrm{n} & =\text { Depends on the transition type } \\
\mathrm{E} & =\text { The energy of incident photon } \\
\mathrm{E}_{\mathrm{g}} & =\text { The optical gap }
\end{array}
$$

The plots of $(\alpha \mathrm{E})^{2}$ Versus the photon Energy (E) showed a linear behavior for $\mathrm{n} 1 / 2$, Fig. 8. This can be taken as evidence that direct transitions occurred in these systems (Mansour, 2004). The $\mathrm{E}_{\mathrm{g}}$ values of the investigated samples were determined from the extrapolation of the straight line to zero photon energy and are listed in Table 1 . These values indicate that there is no significant variation in the optical band gap by increasing the dye content or exposure time which refers to the high stability of this dye.

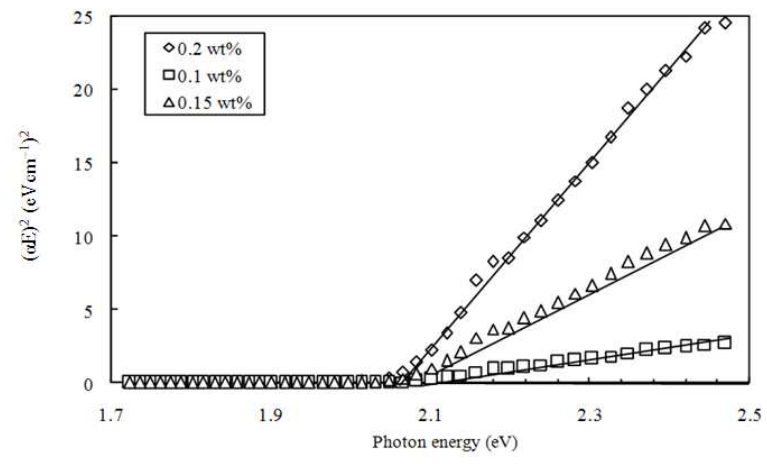

Fig. 8: The dependence of $(\alpha E)^{2}$ on photon energy for various concentrations of Ponceau $2 \mathrm{R}$ in sol-gel glass films

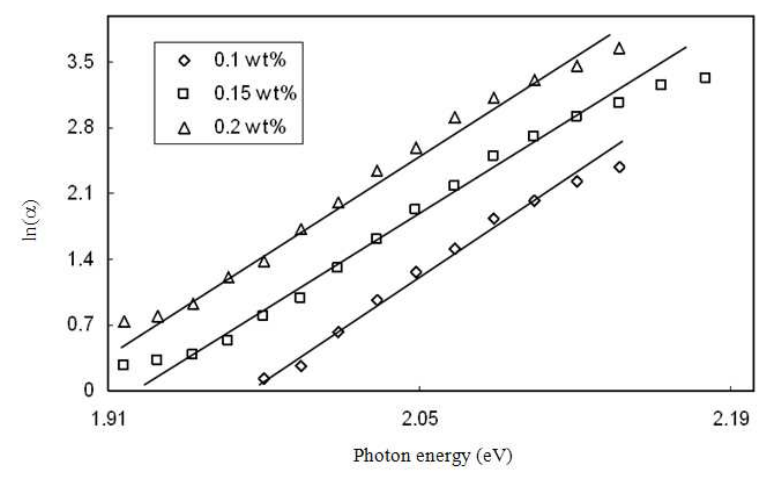

Fig. 9: The variation of $\ln (\alpha)$ Vs photon energy for various concentrations of Ponceau 2R in sol-gel glass films
The dependence of the absorption coefficient on the photon Energy (E) follows the Urbach rule (Mansour, 2004):

$\alpha(h v)=\alpha_{o}(h v) \exp \left(E / E_{u}\right)$

Where:

$\alpha_{\mathrm{o}}=$ A constant

$E_{u}=$ The energy width of the tail of the localized state in the forbidden band gap

Figure 9 presents the Urbach plot of $\ln (\alpha)$ versus Vs photon Energy (E) for different concentrations of ponceau $2 R$. The values of $E_{u}$ were estimated from the slopes of the linear portions of these curves and listed in Table 1. The high value of $E_{u}$ at large dye content may be attributed to a certain structure occurring in the system.

The dependence of $(\alpha E)^{2}$ and/or $\ln (\alpha)$ on the photon energy (E) for our samples after exposure to sunlight were also studied. The results are summarized and listed in Table 1. These data show that there is no variation in the values of $E_{g}$ by increasing exposure times up to 60 days. The values of $E_{u}$ were found to be lower than those for the samples before exposure to sun light. This may be attributed to the nature of dye structure. From the above results we can say that the Ponceau $2 \mathrm{R}$ dye concentrators system has a higher stability for sunlight compared with the other systems (Abbas et al., 2005).

Fluorescence properties: In order to give more information on the efficiency of the solar collectors for our investigated samples the fluorescence properties of these LSCs were investigated. Figure 10 shows the electronic emission spectra for Ponceau $2 \mathrm{R}$ in ethanol solution and sol-gel matrix at excitation wavelength 490 and $453 \mathrm{~nm}$, respectively. In the case of solid matrix, the emission spectra presented a red shift to longer wavelength if compared to the dye in ethanol.

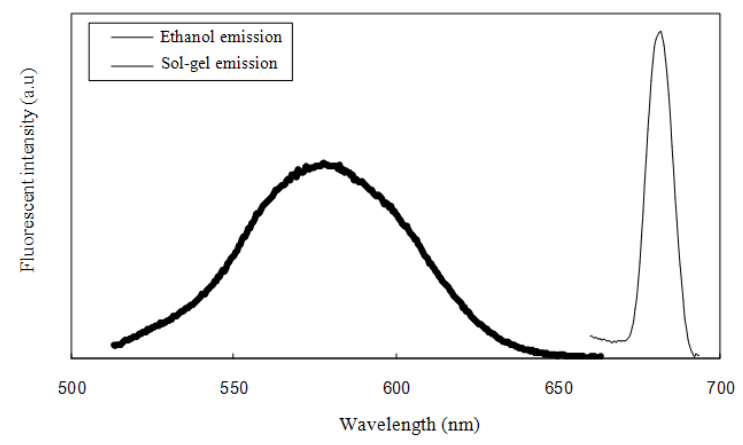

Fig. 10: The fluorescent spectra of Ponceau $2 \mathrm{R}$ in ethanol and the doped sol-gel glass film 


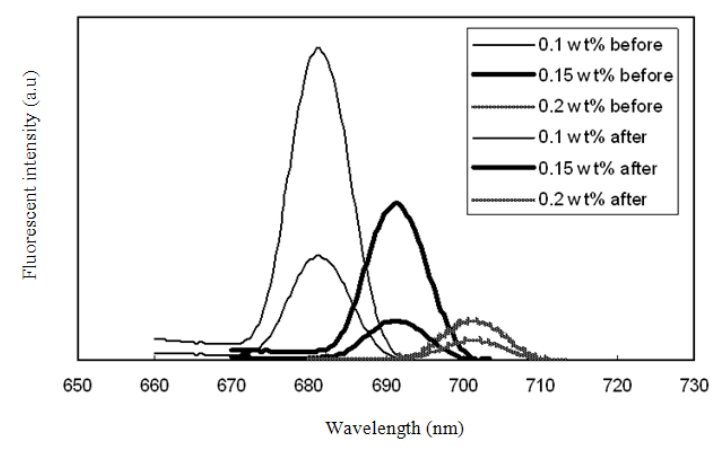

Fig. 11: The fluorescent spectra for various concentrations of Ponceau 2R in sol-gel glass films before and after exposure to sunlight

Table 2: The Stokes shift $(\Delta \lambda)$ and fluorescence Quantum yield $\left(\mathrm{Q}_{\mathrm{f}}\right)$ for Ponceau 2R samples in ethanol solution and in sol-gel films

\begin{tabular}{|c|c|c|c|c|c|}
\hline Samples & $\begin{array}{l}\text { Exposure } \\
\text { time (day) }\end{array}$ & $\begin{array}{l}{ }^{\mathrm{a}} \lambda_{\mathrm{abs}} \\
(\mathrm{nm})\end{array}$ & $\begin{array}{l}{ }^{\mathrm{b}} \boldsymbol{\lambda}_{\mathrm{em}} \\
(\mathrm{nm})\end{array}$ & $\begin{array}{l}\Delta \lambda \\
(\mathrm{nm})\end{array}$ & $Q_{f}$ \\
\hline \multirow[t]{2}{*}{0.10} & 0 & 485 & 682 & 197 & 0.17 \\
\hline & 60 & 485 & 682 & 197 & \\
\hline \multirow[t]{2}{*}{0.15} & 0 & 485 & 693 & 208 & 0.20 \\
\hline & 60 & 485 & 693 & 208 & \\
\hline \multirow[t]{2}{*}{0.20} & 0 & 485 & 702 & 217 & 0.08 \\
\hline & 60 & 485 & 702 & 217 & \\
\hline $\begin{array}{l}\text { In ethanol } \\
\text { solution }\end{array}$ & -- & 502 & 580 & 78 & 0.50 \\
\hline
\end{tabular}

This can be explained on the basis of medium polarity (Hungerford et al., 1999). The silica-gel glass is a porous solid, with a pore shape similar to an ink bottle, which restricts the evaporation of the solvent (Abbas et al., 2005). Owing to the uncompleted evaporation of ethanol the dye molecule stated to interact with the ethanol. Moreover, these pores are richer in isolated $\mathrm{OH}$ groups (Abbas et al., 2005). Thus the environment inside a pore is polar than in ethanol. This explains why the fluorescence peak position of Ponceau $2 R$ is at a longer wavelength than ethanol solution. A change in the intensity of the fluorescent peak is also observed suggesting that only one form of the dye exists in the solid host and ethanol. The fluorescence spectrum for sol-gel doped samples displays a shift to longer wavelength with increasing concentration, Fig. 11 . The origin of the shift has been attributed to aggregate formation (Hungerford et al., 1999), in which a greater number of dye molecules have to occupy a more silicalike environment.

The Stokes shift $(\Delta \lambda)$, which is the difference between the wavelength at which the absorption and emission maxima was observed, was calculated and listed in Table 2. It was observed that the Stokes shift of the Ponceau $2 \mathrm{R}$ in solid matrix is more than that in the liquid medium. This may be due to the difference in environment between solid matrix and solution (Sukumaran and Ramalingam, 2006). Also we notice that, increasing the concentration of Ponceau 2R was accompanied by an increase in the Stokes shift. This indicates low self-absorption of emitted radiation by dye molecules.

The fluorescence quantum yield $\left(\mathrm{Q}_{\mathrm{f}}\right)$ for the investigated samples were measured relative to fluorescein dye as a standard material $\left(\mathrm{Q}_{\mathrm{f}}=0.9\right)$ before exposure to sunlight and calculated using relationship (Reda, 2007):

$$
Q_{f}=Q_{\text {ref }} \times \frac{a_{\text {ref }}}{a_{\text {sam }}} \times \frac{A_{\text {smp }}}{A_{\text {ref }}} \times\left(\frac{n_{\text {sam }}}{n_{\text {ref }}}\right)^{2}
$$

Where:

$$
\begin{aligned}
\mathrm{A}_{\text {sam }} \text { and } \mathrm{A}_{\text {ref }}= & \text { The areas under the fluorescence } \\
& \text { spectrum } \\
\mathrm{a}_{\text {sam }} \text { and } \mathrm{a}_{\mathrm{ref}}= & \text { The absorbance at excitation } \\
& \text { wavelength } \\
\mathrm{n}_{\text {sam }} \text { and } \mathrm{n}_{\mathrm{ref}}= & \text { The refractive indices of the solvent } \\
& \text { and reference respectively }
\end{aligned}
$$

The results obtained are listed in Table 2. Table 2 reveals that the fluorescence quantum yield of the dye in the solid matrix is less than that in the liquid medium. This decrease in the quantum yield may be due to the re-absorption process. Also a chromophore dissolved in a solid matrix is always influenced by its host. Hence, the properties of a chromophore in a solgel matrix, when excited may be affected by its interaction with the silica during the deactivation process (Sukumaran and Ramalingam, 2006). It was also notice that the sample of concentration $(0.1 \mathrm{wt} \%)$ has the highest fluorescent intensity and fluorescence quantum yield. Therefore, this concentration has been selected for field performance of luminescent solar collectors.

The fluorescent properties of our samples after exposure to sunlight for 60 days were also studied and shown in Fig. 11. It was found that the fluorescent intensity decreased as the exposure time increase. This may be attributed to the degradation of the dye due to absorption of solar radiation. The fluorescent parameters of the Ponceau 2R after exposure to sunlight for 60 days at different concentrations were also calculated and tabulated in Table 2. It was found that there was no variation in the values of Stokes shift which reflects that there was only one form of the dye and indicates its high stability (Hungerford et al., 1999). Therefore these samples can be selected for field performance of fluorescent solar collectors. 


\section{CONCLUSION}

Thin-films containing Ponceau $2 \mathrm{R}$ with different concentrations have been prepared by sol-gel spin coating and investigated by spectroscopic techniques. The absorption spectra of the dye doped sol-gel samples differ from those obtained from liquid solution. The observed changes upon incorporation in solid matrix may be attributed to the influence of the host on the dye agglomeration. The photostability of Ponceau $2 \mathrm{R}$ doped sol-gel silica samples through 60 days exposure to sunlight were studied. It was found that the concentration $0.1 \mathrm{wt} \%$ has the lowest degradation rate. The obtained results suggest also that the transition energy for electrons are direct allowed transitions with $\mathrm{n}=1 / 2$ for all examined concentrations. There is no variation in the values of optical gap energy by increasing the dye content and exposure times, while the values of the tail width of the localized states in the band gap vary with concentrations of the dye and exposure times. The florescence spectra of Ponceau $2 \mathrm{R}$ in solid matrix show a red shift compared with solution due to the polar nature of the silica pore. The fluorescence spectrum for sol-gel doped samples show also a dramatic red shift with increasing dye concentration due to aggregate formation. The sample of concentration $0.1 \mathrm{wt} \%$ has the highest fluorescent intensity and fluorescence quantum yield. Therefore, this concentration has been selected for field performance of luminescent solar collectors. So, we can say that our study is useful in preparing luminescent solar collector of this dye using silica gel.

\section{ACKNOWLEDGEMENT}

The researcher is very grateful to the physics department, national research center for cooperation and support.

\section{REFERENCES}

Abbas, H., K.P. Tiwary, L.S.S. Singh, M. Zulfequar and Z.H. Zaidi et al., 2005. Spectroscopic study of sulforhodamine 640-doped sol-gel silica. J. Luminescence, 114: 162-166. DOI: 10.1016/j.jlumin.2004.12.013

Bailey, S.T., G.E. Lokey, M.S. Hanes, J.D.M. Shearer and J.B. McLafferty et al., 2007. Optimized excitation energy transfer in a three-dye luminescent solar concentrator. Solar Energy Mater. Solar Cells, 91: 67-75. DOI: 10.1016/j.solmat.2007.05.001

Costa, T.M.H., V. Stefani, M.R. Gallas, N.M. Balzaretti and J.A.H. Da Jornada, 2004. Fluorescent compacts prepared by the entrapment of benzoxazole type dyes into a silica matrix at high pressure. J. Non-Crystal. Solids, 333: 221-225.
Debije, M.G., J.P. Teunissen, M.J. Kastelijn and P.P.C. Verbunt et al., 2009. The effect of a scatterung layer on the edge output of a luminescent solar concentrator. Solar Energy Material and Solar Cells, 93: 1345-1350. DOI: 10.1016/j.solmat.2009.02.013.

Earp, A.A., G.B. Smith, J. Franklin and P. Swift, 2004. Optimization of a three-color luminescent solar concentrator daylighting system. Solar Energy Mater. Solar Cells, 84: 411-426. DOI: 10.1016/j.solmat.2004.02.046.

Gallagher, S.J., B. Norton and P.C. Eames, 2007. Quantum dot solar concentrators: Electrical efficiencies and comparative concentrating factors of fabricated devices. Solar Energy, 81: 540-547. DOI: 10.1016/j.solener.2006.09.011

Gnanamani, A., M. Bhaskar, R. Ganeshjeevan, R. Chandrasekar and G. Sekaran et al., 2005. Enzymatic and chemical catalysis of xylidine ponceau $2 \mathrm{R}$ and evaluation of products released. Process Biochem., 40: 3497-3504. DOI: 10.1016/j.procbio.2005.03.053

Hungerford, G., K. Suhling and J.A. Ferreira, 1999. Comparison of the fluorescence behavior of rhodamine $6 \mathrm{G}$ in bulk and thin film tetraethylorthosilicate derived sol-gel matrices. J. Photochem. Photobiol. Chem., 129: 71-80.

Laranjo, M.T., V. Stefani, E.V. Benvenutti, T.M.H. Costa and G.D.O. Ramminger et al., 2007. Synthesis of ormosil silica/rhodamine 6G: Powders and compacts. J. Non-Crystal. Solids, 353: 24-30. DOI: 10.1016/j.jnoncrysol.2006.09.029

Mansour, A.F., 2004. Photostability and optical parameters of copolymer styrene/MMA as a matrix for the dyes used in fluorescent solar collectors. Polymer Test., 23: 247-252. DOI: 10.1016/j.polymertesting.2003.09.010.

Maruszewski, K., 1999. Spectroscopic properties of pure and coumarin 153-doped thin films of sol-gel silica xerogels. J Mol. Struct., 479: 53-58.

Pettit, R.B. and C.J. Brinker, 1986. Use of sol-gel thin films in solar energy applications. Solar Energy Mater., 14: 269-287.

Reda, S.M., 2007. Stability and photodegradation of phthalocyanines and hematoporphyrin doped PMMA as solar concentrators. Solar Energy, 81: 755-760. DOI: $10.1016 / j$.solener.2006.10.004

Reisfeld, R., D. Shamrakov and C. Jorgensen, 1994. Photostable solar concentrators based on fluorescent glass films. Solar Energy Mater. Solar Cells, 33: 417-427.

Sukumaran, V.S. and A. Ramalingam, 2006. Spectra characteristics and nonlinear studies of crystal violet dye. Spectrochim. Acta Part Mol. Biomol., 63: 673-676. DOI: 10.1016/j.saa.2005.06.017 